\title{
Nephrotoxicity of cisplatin combination chemotherapy in thoracic malignancy patients with CKD risk factors
}

\author{
Ko Sato ${ }^{1}$, Satoshi Watanabe ${ }^{2 *}$, Aya Ohtsubo ${ }^{1}$, Satoshi Shoji', Daisuke Ishikawa', Tomohiro Tanaka', \\ Koichiro Nozaki ${ }^{1}$, Rie Kondo ${ }^{1}$, Masaaki Okajima', Satoru Miura', Junta Tanaka ${ }^{3}$, Takuro Sakagami ${ }^{1}$, Toshiyuki Koya', \\ Hiroshi Kagamu', Hirohisa Yoshizawa² and Ichiei Narita'
}

\begin{abstract}
Background: Nephrotoxicity is the major side effect that limits the dose of cisplatin that can be safely administered, and it is a clinical problem in cancer patients who received cisplatin combination chemotherapy. Recent evidence has demonstrated that patients with chronic kidney disease (CKD) have an increased risk of developing acute kidney injury (AKI). The present study was conducted to evaluate the prevalence of CKD risk factors in patients who received cisplatin and to assess the correlation between CKD risk factors and cisplatin-induced AKI.

Methods: We retrospectively analyzed 84 patients treated with cisplatin combination chemotherapy for thoracic malignancies. AKI was defined as a decrease in the estimated glomerular filtration rate (eGFR) $>25 \%$ from base line, an increase in the serum creatinine (sCre) level of $>0.3 \mathrm{mg} / \mathrm{dl}$ or $\geq 1.5$ times the baseline level.

Results: Eighty of the 84 patients (95.2\%) had at least one risk factor for CKD. All enrolled patients received cisplatin with hydration, magnesium supplementation and mannitol. Cisplatin-induced AKI was observed in 18 patients (21.4\%). Univariate analysis revealed that cardiac disease and use of non-steroidal anti-inflammatory drugs (NSAIDs) were associated with cisplatin-induced nephrotoxicity (odds ratios [OR] 6 and 3.56, $95 \%$ confidence intervals [CI] 1.21-29.87 and 1.11-11.39, $p=0.04$ and $p=0.04$, respectively). Multivariate analysis revealed that cisplatin nephrotoxicity occurred significantly more often in patients with both risk factors (OR 13.64, $95 \% \mathrm{Cl} 1.11-326.83, p=0.04)$. Patients with more risk factors for CKD tended to have a greater risk of developing cisplatin-induced AKI.
\end{abstract}

Conclusions: We should consider avoiding administration of cisplatin to patients with CKD risk factors, particularly cardiac disease and NSAID use.

Keywords: Cisplatin, Nephrotoxicity, Chronic kidney disease, Acute kidney injury

\section{Background}

Cisplatin is one of the most commonly administered agents in the treatment of cancer. Cisplatin-based chemotherapy has demonstrated survival benefits for patients with resected early-stage non-small cell lung cancer (NSCLC), advanced-stage NSCLC, small-cell lung cancer (SCLC) and malignant pleural mesothelioma [1-4]. However, cisplatin can have severe side effects-in particular, cisplatin-induced nephrotoxicity, which is dose

\footnotetext{
*Correspondence: satoshi7@med.niigata-u.ac.jp

${ }^{2}$ Bioscience Medical Research Center, Niigata University Medical and Dental Hospital, Niigata City, Niigata, Japan

Full list of author information is available at the end of the article
}

dependent and is the major side effect that limits the acceptable dose of cisplatin [5]. Impairment of renal function has been shown to be a clinical problem in 25-35\% of patients treated with cisplatin [5]. Therefore, it is important to identify the predictors of cisplatin-induced nephrotoxicity and to avoid administrating cisplatin to patients with these risk factors.

Recent studies have reported several risk factors for chronic kidney disease (CKD), including lifestyle-related diseases, smoking, use of non-steroidal anti-inflammatory drugs (NSAIDs), cardiac disease and cerebrovascular disease [6]. Patients with risk factors for CKD may have a higher incidence of cisplatin-induced nephrotoxicity. 
Indeed, acute kidney injury (AKI) is more frequently observed in patients with CKD [7, 8]. Furthermore, recent studies have reported that patients with lung cancer have a higher incidence of comorbidities than do patients with other cancers [9]. The aims of this study were to evaluate the prevalence of CKD risk factors in thoracic malignancy patients who received cisplatin and to elucidate the correlation between nephrotoxicity due to cisplatin-based chemotherapy in patients with CKD risk factors.

\section{Methods}

\section{Patients}

We retrospectively analyzed the data of consecutive patients who received cisplatin combination chemotherapy for thoracic malignancies as the first-line chemotherapy in our hospital between January 2006 and December 2012. All patients provided written informed consent. The study was conducted in accordance with the Helsinki Declaration of the World Medical Association. The protocol was approved by the institutional review board of Niigata University.

\section{Hydration and treatment methods}

Patients received cisplatin combination chemotherapy every 3-4 weeks for 4-6 cycles. As a common antiemetic premedication, granisetron $(3 \mathrm{mg})$ or palonosetron $(0.75 \mathrm{mg})$ was combined with dexamethasone $(9.9 \mathrm{mg}$ ) and dissolved in $100 \mathrm{ml}$ of normal saline and infused to the patient, and oral aprepitant $(125 \mathrm{mg}$ on day1, $80 \mathrm{mg}$ on days 2-3) was given before and after chemotherapy. Cisplatin was administered in $250 \mathrm{~mL}$ of normal saline solution over $2 \mathrm{~h}$ in combination with other chemotherapeutic agents and $3000-3850 \mathrm{ml}$ of hydration. All patients received $40 \mathrm{mEq}$ of magnesium sulfate and $300 \mathrm{ml}$ of mannitol to prevent nephrotoxicity (Additional file 1: Figure S1).

\section{Nephrotoxicity evaluation}

The estimated glomerular filtration rate (eGFR) $(\mathrm{mL} / \mathrm{min} /$ $1.73 \mathrm{~m}^{2}$ ) was calculated using the equation of the Japanese Society of Nephrology: eGFR $\left(\mathrm{mL} / \mathrm{min} / 1.73 \mathrm{~m}^{2}\right)=194 \mathrm{x}$ serum creatinine $(\mathrm{sCre})^{-1.094} \times$ age $^{-0.287}(\times 0.739$ if female $)$ [10]. We calculated and recorded the eGFR at four time points: before the administration of cisplatin combination chemotherapy, the minimum value during chemotherapy, 4 weeks after the last administration of cisplatin and the maximum value during the follow-up period (6 months after the last administration of cisplatin). AKI was defined as follows: a decrease in eGFR $>25 \%$ of baseline, an increase in sCre level of $>0.3 \mathrm{mg} / \mathrm{dl}$ or $\geq 1.5$ times the baseline level in reference to the RIFLE (risk, injury, failure, loss, and end stage) criteria and the Common Terminology Criteria for Adverse Events version $4.0[11,12]$.

\section{Risk factors}

To identify the risk factors potentially associated with the occurrence of a cisplatin-induced nephrotoxicity, we examined the relationship between AKI incidence and the following 17 CKD risk factors: old age ( $\geq 70$ years old), proteinuria, hematuria, dyslipidemia, diabetes mellitus, hypertension, hyperuricemia, obesity (body mass index $\geq 25$ ), smoking, NSAID use, cerebrovascular disease, cardiac disease, autoimmune disease, infectious disease, urinary-tract stone and a history of acute renal failure [13]. We also assessed the following potential risk factors: hypoalbuminemia (serum albumin level $<3.5 \mathrm{~g} / \mathrm{dL}$ ), Eastern Cooperative Oncology Group performance status (PS $\geq 1$ ), the number of CKD risk factors, sex, Charlson Comorbidity Index (CCI) score, and combined anticancer drugs [14-16].

\section{Statistical analysis}

The differences in the categorical variables between patients with and those without AKI were determined using the chi-squire test or Fisher's exact test, as appropriate. Two-sided $P<0.05$ was considered statistically significant. To identify the variables significantly associated with AKI, multivariate logistic regression analysis was performed. All statistical analyses were performed using the JMP 9.0.2 statistical software (SAS Institute, Cary, NC, USA).

\section{Results}

\section{Patient characteristics}

A total of 84 patients who were treated with cisplatin combination chemotherapy for thoracic malignancies were included in this study. The patient characteristics are listed in Table 1. There were 19 (22.6 \%) female patients and the median age of all the patients was 61 years old (range, 33-75 years). Seventy-nine (94\%) patients had a PS 0-1, 52 (61.9\%) had NSCLC and 19 (22.6\%) had SCLC. The median cisplatin dosage was $80 \mathrm{mg} / \mathrm{m}^{2} /$ day, and the median number of cycles was 4 (range, 1-6). The patients received various cisplatin combination drugs, including vinorelbine in 26 (31\%), etoposide in 14 (16.7 \%), docetaxel in $12(14.3 \%)$, pemetrexed in 11 (13.1\%), gemcitabine in 4 (4.8\%), S-1 in $4 .(4.8 \%)$, irinotecan in $2(2.4 \%)$, and others in $11(13.1 \%)$. The median CCI was 6 (range, 2-10).

\section{Incidence and outcome of AKI}

Cisplatin-induced AKI was observed in 18 (21.4\%) of the 84 enrolled patients (Additional file 2: Table S1). In the AKI group and in the no AKI group, the median eGFR before administration of cisplatin was 79.2 (range, 59.2-101) and 82.4 (range, 60-113) $\mathrm{mL} / \mathrm{min} /$ $1.73 \mathrm{~m}^{2}$, respectively. Two patients had mild renal dysfunction before cisplatin administration (eGFR, 59.2 
Table 1 Base line characteristics of all study patients $(n=84)$

\begin{tabular}{|c|c|}
\hline Parameter & \\
\hline \multicolumn{2}{|l|}{ Gender } \\
\hline Male & $65(77.3 \%)$ \\
\hline Female & 19 (22.6 \%) \\
\hline \multicolumn{2}{|l|}{ Age (y) } \\
\hline Median (Range) & $61(33-75)$ \\
\hline \multicolumn{2}{|l|}{ Performance status } \\
\hline $0 / 1 / 2$ & $41 / 38 / 0$ \\
\hline 3/4/unknown & $1 / 0 / 4$ \\
\hline \multicolumn{2}{|l|}{ Histology } \\
\hline Small cell carcinoma & 19 (22.6 \%) \\
\hline Adenocarcinoma & $37(44 \%)$ \\
\hline Large-cell carcinoma & $2(2.4 \%)$ \\
\hline Squamous cell carcinoma & $13(15.5 \%)$ \\
\hline Malignant pleural mesothelioma & $6(7.1 \%)$ \\
\hline Thymic malignancy & $2(2.4 \%)$ \\
\hline Others & $5(6 \%)$ \\
\hline \multicolumn{2}{|l|}{ Cisplatin } \\
\hline Median dose (mg/m²/day) (range) & $80(40-80)$ \\
\hline Median cycle (range) & $4(1-6)$ \\
\hline \multicolumn{2}{|l|}{ Anticancer drugs } \\
\hline Vinorelbine & $26(31 \%)$ \\
\hline Etoposide & 14 (16.7 \%) \\
\hline Docetaxel & $12(14.3 \%)$ \\
\hline Pemetrexed & $11(13.1 \%)$ \\
\hline Gemcitabine & $4(4.8 \%)$ \\
\hline S-1 & $4(4.8 \%)$ \\
\hline Irinotecan & 2 (2.4\%) \\
\hline Others & $11(13.1 \%)$ \\
\hline \multicolumn{2}{|l|}{ Serum creatinine (mg/dl) } \\
\hline Median (range) & $0.72(0.50-0.99)$ \\
\hline \multicolumn{2}{|l|}{$\mathrm{eGFR}\left(\mathrm{mL} / \mathrm{min} / 1.73 \mathrm{~m}^{2}\right)$} \\
\hline Median (range) & $81.1(59.2-113.0)$ \\
\hline \multicolumn{2}{|l|}{ Charlson Comorbidity Index } \\
\hline Median (range) & $6(2-10)$ \\
\hline
\end{tabular}

and $59.6 \mathrm{~mL} / \mathrm{min} / 1.73 \mathrm{~m}^{2}$ ). Additional file 2: Table S1 shows the number of patients with risk factors for CKD in the AKI group and the no AKI group. In both groups, the most frequent risk factor was smoking (77.8 \% in the patients with AKI and $83.3 \%$ in the patients without AKI). All 18 (100\%) of the patients with AKI and 61 (92.4\%) of the patients without AKI had at least one risk factor.

Figure 1 shows the changes in eGFR in the 18 patients with AKI. During follow-up period (6 months after the last administration of cisplatin), the eGFR in 5 patients
(27.8\%) recovered to baseline, the eGFR in 11 patients (61.1\%) recovered to between $75 \%$ and baseline, and the eGFR in 2 patients $(11.1 \%)$ did not recover to $75 \%$ of baseline.

\section{Risk factors for developing AKI}

In the univariate analysis, we identified cardiac disease (odds ratio [OR] 6, 95 \% confidence interval [CI] 1.2129.87, $p=0.04$ ) and NSAID use (OR 3.56, $95 \% \mathrm{CI}$ $1.11-11.39, p=0.04)$ as significant predictors of AKI (Table 2). The eGFR before treatment $(p=0.22)$, and the other risk factors for CKD, such as PS $\geq 1 \quad(p=0.48)$, hypoalbuminemia $(p=0.49)$, female sex $(p=0.56)$, CCI score $(p=0.78)$, the type of anticancer drugs $(p=0.15-0.9)$ and the number of treatment cycles $(p=0.74)$ were not significantly associated with cisplatin-induced AKI. Next, we included cardiac disease and NSAID use in the logistic regression model with the addition of age and sex to assess the contribution of each risk factor to cisplatin-induced AKI. Although each of these factors was not a significant predictor of cisplatin-induced AKI, we found that cardiac disease and NSAID use had relatively higher odds ratios (OR 5, 95 \% CI 0.89-30.4 and OR 3.45, 95 \% CI 0.9712.12, respectively; Table 3). Additionally, AKI occurred significantly more often in patients who presented both cardiac disease and NSAID use (OR 13.64, 95 \% CI 1.11326.83, $p=0.04$ ). The multivariate analyses also revealed that the patients with more risk factors for CKD tended to have an increased risk of developing cisplatin-induced AKI.

\section{Discussion}

Cisplatin is one of the most active cytotoxic agents in treatment of thoracic malignancies. Cisplatin or carboplatin combination chemotherapy is recommended as the first-line chemotherapy for patients with lung cancer $[17,18]$. Because cisplatin has more severe nonhematological toxicity than carboplatin does, particularly in terms of nephrotoxicity and gastrointestinal toxicity, cisplatin is more poorly tolerated than carboplatin. Although cisplatin-based chemotherapy has been reported to be superior to carboplatin-based chemotherapy in non-squamous NSCLC patients, the number of patients administered cisplatin-based chemotherapy was limited in clinical practice [2]. Recent progress in antiemetic therapies has greatly reduced cisplatin-induced gastrointestinal toxicity. We and other investigators have reported that triple antiemetic therapy with a $5-\mathrm{HT}_{3}$ receptor antagonist, dexamethasone and aprepitant ameliorates cisplatin-induced nausea and vomiting [19, 20]. However, cisplatin-induced nephrotoxicity remains an unsolved problem. Although previous studies have reported less toxic methods for administrating cisplatin, such as hydration with the supplementation of magnesium and mannitol, cisplatin-induced nephrotoxicity 


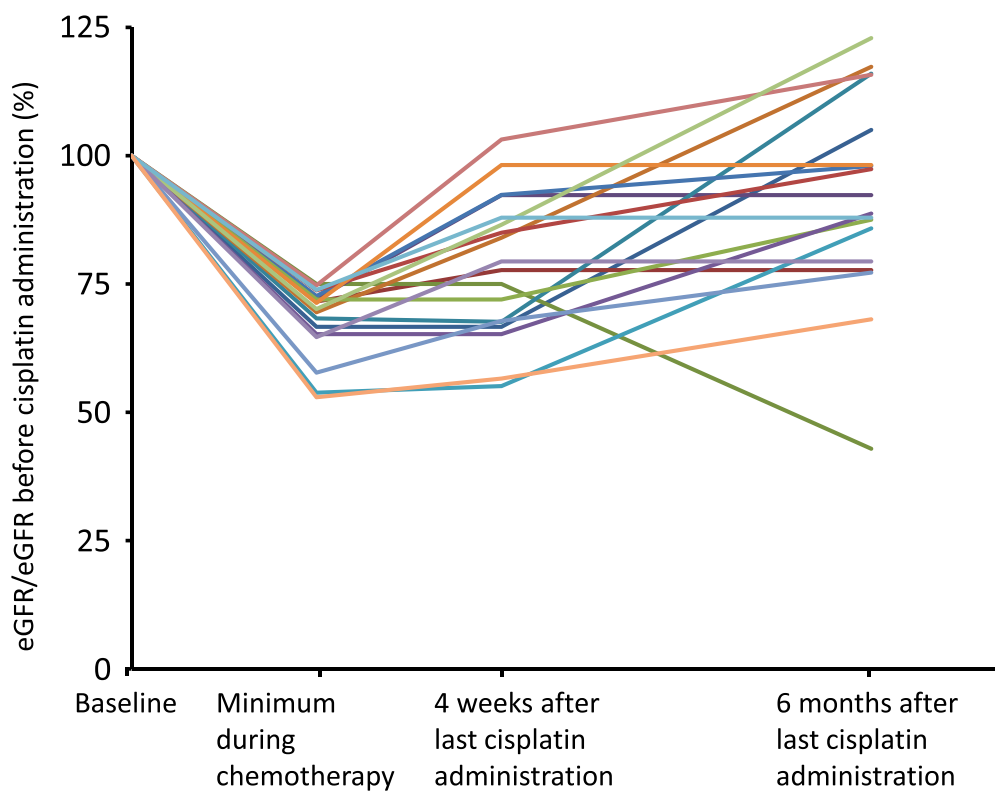

Fig. 1 Changes of estimated glomerular filtration rate (eGFR). The eGFR was measured at four time points; before the treatment, under treatment, 4 weeks after last cisplatin administration and during follow-up period (6 months after last cisplatin administration)

still occurs in $6-14 \%$ of patients [21, 22]. Compatible with these studies, the current study showed that the incidence of AKI was $21.4 \%(18 / 84)$, despite the administration of hydration, magnesium supplementation and mannitol. We need to develop more effective strategies to prevent cisplatin-induced nephrotoxicity.

Previous studies demonstrated that old age, female sex, current smoking, hyperuricemia, hypoalbuminemia, diabetes mellitus, cardiovascular disease and NSAID use are risk factors for cisplatin-induced nephrotoxicity $[15,21,23]$. Because recent evidence has demonstrated that CKD is a risk factor for AKI, we hypothesized that patients with risk factors for CKD would also be at risk for cisplatin-induced nephrotoxicity [7]. The present study showed that comorbidities, including CKD risk factors, were frequent in patients with thoracic malignancies (Additional file 2: Table S1). Univariate analysis demonstrated that cardiac disease and NSAID use were statistically significant predictors of cisplatin-induced nephrotoxicity (Table 2). Although multivariate analysis revealed that no single factor was significantly associated with cisplatin-induced AKI, AKI did occur significantly more frequently in patients who had both cardiac disease and NSAID use (Table 3). The risk factors for cardiac disease, such as hypertension, diabetes mellitus, dyslipidemia and smoking, are also risk factors for CKD. These factors cause atherosclerosis in the renal arteries and induce chronic ischemic injury to the tubulointerstitium [24]. Because tubular cells are the major target of cisplatin-induced nephrotoxicity, it is not surprising that cardiac disease is associated with this complication [25]. Nonselective cyclooxygenase (COX) inhibition by NSAIDs decreases prostaglandin synthesis and induces vasoconstriction and renal ischemia. Cisplatin has been suggested to induce injury in the renal vasculature and ischemic injury of the kidneys; thus these effects of NSAIDs may exacerbate cisplatin-induced nephrotoxicity [25]. In this study, the non-selective COX inhibitor loxoprofen was prescribed to 13 of the 17 patients who had received NSAIDs (76.8 \%). Use of selective COX 2 inhibitors may reduce the risk of cisplatin-induced nephrotoxicity. Furthermore, our study showed that an increased number of CKD risk factors may be related to a higher incidence of cisplatin-induced AKI (Tables 2 and 3). Patients without CKD risk factors did not develop cisplatin-induced AKI. By contrast, patients with 5 or more CKD risk factors had relatively higher odds ratio (OR 4.55, 95 \% CI $0.73-$ 28.65; Table 3). We also investigated whether CCI score, which is a method of classifying prognostic comorbidity, correlated with the incidence of cisplatin-induced AKI, but no association was found [16].

In previous studies, cisplatin-induced nephrotoxicity has been assessed on the basis of the elevated sCre levels $[21,26]$. However, Launay-Vacher et al. analyzed data for 4684 cancer patients and found that elevated sCre levels were less sensitive than was decreased Cre clearance for detecting renal impairment [27]. In that study, renal impairment was commonly found in cancer patients (57.4\%), and $53.4 \%$ required dose reduction due to nephrotoxicity. The authors concluded that the use of 
Table 2 Univariate analysis by chi-square test or Fisher's exact test

\begin{tabular}{|c|c|c|c|}
\hline Risk factor & Odds ratio & $95 \%$ C.I. & $P$-value \\
\hline \multicolumn{4}{|l|}{ CKD risk factors } \\
\hline Smoking & 0.7 & $0.19-2.53$ & 0.59 \\
\hline Hypertension & 2.3 & $0.79-6.66$ & 0.12 \\
\hline Dyslipidemia & 2.25 & $0.70-7.20$ & 0.17 \\
\hline Use of NSAIDs & 3.56 & $1.11-11.39$ & 0.04 \\
\hline Obesity & 0.51 & $0.10-2.50$ & 0.4 \\
\hline Diabetes mellitus & 0.91 & $0.17-4.70$ & 0.91 \\
\hline Hyperuricemia & 1.05 & $0.20-5.57$ & 0.95 \\
\hline Urinary tract stone & - & & 0.12 \\
\hline Age $\geq 70$ & 0.5 & $0.06-4.31$ & 0.52 \\
\hline Cardiac disease & 6 & $1.21-29.87$ & 0.04 \\
\hline Hematuria & - & & 0.29 \\
\hline Cerebrovascular disease & - & & 0.29 \\
\hline Autoimmune disease & 4 & $0.52-30.61$ & 0.15 \\
\hline Proteinuria & - & & 0.6 \\
\hline Infectious disease & - & & - \\
\hline Past history of ARF & - & & - \\
\hline Pt. with CKD risk & - & & 0.29 \\
\hline Pt. without CKD risk & - & & 0.29 \\
\hline \multicolumn{4}{|l|}{ No. of CKD risk factors } \\
\hline$\leq 2$ & 1 & & \\
\hline 3 & 1.54 & $0.39-5.96$ & 0.72 \\
\hline 4 & 1.5 & $0.34-6.7$ & 0.69 \\
\hline$\geq 5$ & 5 & $0.85-29.39$ & 0.09 \\
\hline \multicolumn{4}{|l|}{ Other risk factors } \\
\hline$P S \geq 1$ & 0.68 & $0.23-2.01$ & 0.48 \\
\hline Alb $<3.5 \mathrm{~g} / \mathrm{dL}$ & 0.69 & $0.24-1.97$ & 0.49 \\
\hline Female & 1.43 & $0.44-4.69$ & 0.56 \\
\hline \multicolumn{4}{|l|}{ Anticancer drugs } \\
\hline Vinorelbine & 1 & $0.35-3.25$ & 0.9 \\
\hline Etoposide & 2.44 & $0.70-8.49$ & 0.15 \\
\hline Docetaxel & 0.7 & $0.14-3.53$ & 0.66 \\
\hline Pemetrexed & 0.33 & $0.04-2.76$ & 0.29 \\
\hline Gemcitabine & 3.94 & $0.51-30.14$ & 0.16 \\
\hline S-1 & 3.94 & $0.51-30.14$ & 0.16 \\
\hline
\end{tabular}

AKI acute kidney injury, CKD chronic kidney disease, NSAID non-steroidal anti-inflammatory drug, ARF acute renal failure, PS performance status, C.I. confidential interval

Bold $P$-values are significant at the 0.05 level

nephrotoxic drugs should be avoided in cancer patients at high risk for AKI. Thus, we included decreased eGFR (estimated using the equation developed by the Japanese Society of Nephrology) in our AKI criteria [10]. The current study demonstrated that a decrease in the eGFR $\geq 25 \%$ from the baseline level was detected in $22.4 \%(18 / 84)$ of the patients, although
Table 3 Multivariate logistic regression analysis adjusted for age and sex

\begin{tabular}{llll}
\hline Parameter & Odds ratio & $95 \%$ C.I. & P-value \\
\hline Age & 1.01 & $0.95-1.08$ & 0.74 \\
Female & 1.99 & $0.52-7.11$ & 0.3 \\
Cardiac disease & 5 & $0.89-30.4$ & 0.07 \\
Use of NSAIDs & 3.45 & $0.97-12.12$ & 0.06 \\
Cardiac disease and use of NSAIDs & 13.64 & $1.11-326.83$ & $\mathbf{0 . 0 4}$ \\
No. of CKD risk factors & & & \\
$\geq 3$ & 2.11 & $0.69-6.79$ & 0.19 \\
$\geq 4$ & 2.68 & $0.83-8.5$ & 0.1 \\
$\geq 5$ & 4.55 & $0.73-28.65$ & 0.1 \\
\hline
\end{tabular}

NSAID non-steroidal anti-inflammatory drug

Bold $P$-value is significant at the 0.05 level

only $9.5 \%(8 / 84)$ exhibited elevated sCre levels of $>0.3 \mathrm{mg} / \mathrm{dl}$ or $\geq 1.5$ times baseline. These data suggest that GFR should be included in assessment of cisplatin-induced nephrotoxicity. Cisplatin causes cell injury and death in the proximal and distal tubules. Although the long-term effects of cisplatin on renal function have not been fully elucidated, previous studies indicated that cisplatin treatment may lead to subclinical but permanent reductions in GFR [28]. By contrast, Moon et al. reported that the elevated sCre levels recovered in $80.5 \%$ of patients after cisplatininduced AKI [29]. In the present study, of the $18 \mathrm{pa}$ tients who had cisplatin-induced AKI, the eGFR in 16 $(88.9 \%)$ recovered to more than $75 \%$ of the baseline level during the follow-up period. Cisplatin combination chemotherapy has been recommended as the first-line chemotherapy for lung cancer patients [17, 18]. Patients who develop cisplatin-induced AKI may have a higher risk of nephrotoxicity with subsequent chemotherapies. Indeed, AKI is a well-defined risk factor for CKD, and patients who develop AKI have a several-fold increased risk of developing CKD [30]. Further investigation should be carried out to evaluate whether subsequent chemotherapies can be safely administered to patients who had experienced cisplatin AKI.

\section{Conclusion}

To our knowledge, this is the first study to assess the incidence of cisplatin-induced nephrotoxicity in patients with CKD risk factors. We identified cardiac disease and NSAID use as risk factors for cisplatin-induced nephrotoxicity. In addition, patients who had a higher number of CKD risk factors tended to have an increased risk of cisplatin-induced nephrotoxicity.

\section{Availability of data and materials}

The dataset supporting the conclusions of this article is included within the Additional file 3: Dataset S1. 


\section{Additional files}

Additional file 1: Figure S1. Chemotherapy hydration protocol used in this study. Cisplatin and other anticancer drugs were administrated with $3850 \mathrm{ml}$ of hydration, magnesium sulfate (40 mEq) and mannitol (300 ml). (PPTX $79 \mathrm{~kb}$ )

Additional file 2: Table S1. The characteristics of the patients with or without AKI. (XLSX 14 kb)

Additional file 3: Dataset S1. The characteristics of the patients. (XLSX $28 \mathrm{~kb}$ )

\section{Abbreviations}

AKI: acute kidney injury; CCl: Charlson Comorbidity Index; CKD: chronic kidney disease; COX: cyclooxygenase; eGFR: estimated glomerular filtration rate; NSAIDs: non-steroidal anti-inflammatory drugs; NSCLC: non-small cell lung cancer; SCLC: small-cell lung cancer; sCre: serum creatinine.

\section{Competing interests}

The authors declare that they have no competing interests.

\section{Authors' contributions}

KS collected the clinical data and drafted the manuscript. SW conducted the study. AO, SS, DI, T, KN, RK, MO and SM participated in patient care and collected the data. JT, TS, TK and HK analyzed and interpreted the data. HY and IN provided the administrative support. All the authors have read and approved the final version of the manuscript.

\section{Acknowledgements}

Special thanks to Kumiko Shirai for her expert assistance with data collection and management.

\section{Author details}

'Department of Medicine (II), Niigata University Medical and Dental Hospital, Niigata City, Niigata, Japan. ${ }^{2}$ Bioscience Medical Research Center, Niigata University Medical and Dental Hospital, Niigata City, Niigata, Japan. ${ }^{3}$ Department of Health Promotion Medicine, Niigata University Medical and Dental Hospital, Niigata City, Niigata, Japan.

\section{Received: 21 January 2015 Accepted: 10 March 2016} Published online: 15 March 2016

\section{References}

1. Pignon JP, Tribodet H, Scagliotti GV, Douillard JY, Shepherd FA, Stephens RJ, Dunant A, Torri V, Rosell R, Seymour L, et al. Lung adjuvant cisplatin evaluation: a pooled analysis by the LACE Collaborative Group. J Clin Oncol. 2008:26(21):3552-9.

2. Ardizzoni A, Boni L, Tiseo M, Fossella FV, Schiller JH, Paesmans M, Radosavljevic D, Paccagnella A, Zatloukal P, Mazzanti P, et al. Cisplatinversus carboplatin-based chemotherapy in first-line treatment of advanced non-small-cell lung cancer: an individual patient data meta-analysis. J Natl Cancer Inst. 2007;99(11):847-57.

3. Rossi A, Martelli O, Di Maio M. Treatment of patients with small-cell lung cancer: from meta-analyses to clinical practice. Cancer Treat Rev. 2013;39(5):498-506.

4. Vogelzang NJ. Phase III Study of Pemetrexed in Combination With Cisplatin Versus Cisplatin Alone in Patients With Malignant Pleural Mesothelioma. J Clin Oncol. 2003;21(14):2636-44.

5. dos Santos NA, Carvalho Rodrigues MA, Martins NM, dos Santos AC. Cisplatin-induced nephrotoxicity and targets of nephroprotection: an update. Arch Toxicol. 2012;86(8):1233-50.

6. Yamagata K, Ishida K, Sairenchi T, Takahashi H, Ohba S, Shiigai T, Narita M, Koyama A. Risk factors for chronic kidney disease in a community-based population: a 10-year follow-up study. Kidney Int. 2007:71(2):159-66.

7. Kidney Disease: Improving Global Outcomes (KDIGO) Acute Kidney Injury Work Group. KDIGO clinical practice guideline for acute kidney injury. Kidney Int Suppl. 2012;2:1-138.

8. Hsu CY, Ordoñez JD, Chertow GM, Fan D, McCulloch CE, Go AS. The risk of acute renal failure in patients with chronic kidney disease. Kidney Int. 2008;74(1):101-7.

9. Edwards BK, Noone A-M, Mariotto AB, Simard EP, Boscoe FP, Henley SJ, Jemal A $\mathrm{Cho} \mathrm{H}$, Anderson RN, Kohler BA, et al. Annual Report to the Nation on the status of cancer, 1975-2010, featuring prevalence of comorbidity and impact on survival among persons with lung, colorectal, breast, or prostate cancer. Cancer. 2014;120(9):1290-314

10. Matsuo S, Imai E, Horio M, Yasuda $Y$, Tomita K, Nitta $K$, Yamagata $K$, Tomino $Y$, Yokoyama H, Hishida A. Revised equations for estimated GFR from serum creatinine in Japan. Am J Kidney Dis. 2009;53(6):982-92.

11. Bellomo R, Ronco C, Kellum JA, Mehta RL, Palevsky P. Acute renal failure definition, outcome measures, animal models, fluid therapy and information technology needs: the Second International Consensus Conference of the Acute Dialysis Quality Initiative (ADQI) Group. Crit Care. 2004;8(4):R204-12.

12. Common Terminology Criteria for Adverse Events v4.0 (CTCAE). 6/2010 update. Available at: http://ctep.cancer.gov/protocolDevelopment/ electronic applications/ctchtm\#ctc 40. Accessed 15 Mar 2016.

13. Li PK, Chow KM, Matsuo S, Yang CW, Jha V, Becker G, Chen N, Sharma SK, Chittinandana A, Chowdhury S, et al. Asian chronic kidney disease best practice recommendations: positional statements for early detection of chronic kidney disease from Asian Forum for Chronic Kidney Disease Initiatives (AFCKDI). Nephrology (Carlton). 2011;16(7):633-41.

14. Oken MM, Creech RH, Tormey DC, Horton J, Davis TE, McFadden ET, Carbone PP. Toxicity and response criteria of the Eastern Cooperative Oncology Group. Am J Clin Oncol. 1982;5(6):649-55.

15. Mathe C, Bohacs A, Duffek L, Lukacsovits J, Komlosi ZI, Szondy K, Horvath I, Muller V, Losonczy G. Cisplatin nephrotoxicity aggravated by cardiovascular disease and diabetes in lung cancer patients. Eur Respir J. 2010;37(4):888-94.

16. Charlson ME, Pompei $P$, Ales $K L$, Mackenzie CR. A new method of classifying prognostic comorbidity in longitudinal studies: development and validation. J Chronic Dis. 1987:40(5):373-83.

17. Goldstraw P, Ball D, Jett JR, Le Chevalier T, Lim E, Nicholson AG, Shepherd FA. Non-small-cell lung cancer. Lancet. 2011;378(9804):1727-40.

18. van Meerbeeck JP, Fennell DA, De Ruysscher DK. Small-cell lung cancer. Lancet. 2011:378(9804):1741-55.

19. Warr DG, Grunberg SM, Gralla RJ, Hesketh PJ, Roila F, Wit R, Carides AD, Taylor A, Evans JK, Horgan KJ. The oral NK(1) antagonist aprepitant for the prevention of acute and delayed chemotherapy-induced nausea and vomiting: Pooled data from 2 randomised, double-blind, placebo controlled trials. Eur J Cancer. 2005;41(9):1278-85.

20. Miura S, Watanabe S, Sato K, Makino M, Kobayashi O, Miyao $H$, Iwashima A, Okajima M, Tanaka J, Tanaka H, et al. The efficacy of triplet antiemetic therapy with $0.75 \mathrm{mg}$ of palonosetron for chemotherapy-induced nausea and vomiting in lung cancer patients receiving highly emetogenic chemotherapy. Support Care Cancer. 2013;21(9):2575-81.

21. Kidera Y, Kawakami H, Sakiyama T, Okamoto K, Tanaka K, Takeda M, Kaneda H, Nishina S, Tsurutani J, Fujiwara K, et al. Risk factors for cisplatin-induced nephrotoxicity and potential of magnesium supplementation for renal protection. PLoS ONE. 2014;9(7):e101902.

22. Muraki K, Koyama R, Honma Y, Yagishita S, Shukuya T, Ohashi R, Takahashi F, Kido K, Iwakami S, Sasaki S, et al. Hydration with magnesium and mannitol without furosemide prevents the nephrotoxicity induced by cisplatin and pemetrexed in patients with advanced non-small cell lung cancer. J Thorac Dis. 2012:4(6):562-8.

23. Lagrange JL, Medecin B, Etienne MC, Pivot X, Cassuto-Viguier E, Renee N, Thyss A, Ferrero JM, Otto J, Francois $E$, et al. Cisplatin nephrotoxicity: a multivariate analysis of potential predisposing factors. Pharmacotherapy. 1997;17(6):1246-53.

24. Fine LG, Norman JT. Chronic hypoxia as a mechanism of progression of chronic kidney diseases: from hypothesis to novel therapeutics. Kidney Int. 2008;74(7):867-72

25. Pabla N, Dong Z. Cisplatin nephrotoxicity: mechanisms and renoprotective strategies. Kidney Int. 2008;73(9):994-1007.

26. Stewart DJ, Dulberg CS, Mikhael NZ, Redmond MD, Montpetit VA, Goel R. Association of cisplatin nephrotoxicity with patient characteristics and cisplatin administration methods. Cancer Chemother Pharmacol. 1997:40(4):293-308.

27. Launay-Vacher V, Oudard S, Janus N, Gligorov J, Pourrat X, Rixe O, Morere J-F, Beuzeboc P, Deray G. Prevalence of Renal Insufficiency in cancer patients and implications for anticancer drug management. Cancer. 2007;110(6):1376-84.

28. Brillet G, Deray G, Jacquiaud C, Mignot L, Bunker D, Meillet D, Raymond F, Jacobs C. Long-term renal effect of cisplatin in man. Am J Nephrol. 1994;14(2):81-4.

29. Moon HH. Prediction of nephrotoxicity induced by cisplatin combination chemotherapy in gastric cancer patients. World J Gastroenterol. 2011; 17(30):3510

30. Leung KCW, Tonelli M, James MT. Chronic kidney disease following acute kidney injury_risk and outcomes. Nat Rev Nephrol. 2012;9(2):77-85. 